\title{
PERFORMANCE OF INTERLOCKING BRICK WALLS AGAINST OUT-OF-PLANE EXCITATION
}

\author{
*Aiko Furukawa ${ }^{1}$, Johanes Jefry Prasetyo ${ }^{2}$ and Junji Kiyono ${ }^{1}$ \\ ${ }^{1}$ Department of Urban Management, Kyoto University, Japan; ${ }^{2}$ Pacific Consultants CO., LTD., Japan
}

*Corresponding Author, Received: 06 Oct. 2021, Revised: 01 Dec. 2021, Accepted: 09 Jan. 2022

\begin{abstract}
Masonry structure is one of the most commonly used building types in developing countries due to its inexpensiveness and material availability. While masonry structure is intrinsically strong in resisting gravitational force, it is relatively weak in resisting lateral forces such as an earthquake. Therefore, reinforcement for masonry structures in earthquake-prone areas is necessary. This paper introduced an interlocking brick as one of the promising reinforcement methods for masonry structure as it does not require extra material and is easy to implement. The performance of a lego-shaped interlocking brick in resisting dynamic lateral forces was investigated through 2-dimensional dynamic finite element analysis. The created lego-shaped interlocking brick wall was analyzed under the out-of-plane dynamic loading. Its performance was compared with the performance of the rectangular brick wall. The analysis results showed that the lego-shaped interlocking brick wall had better performance in resisting out-of-plane loading than the rectangular brick wall since the blocks interlock each other.
\end{abstract}

Keywords: Interlocking brick, Rectangular brick, Out-of-plane excitation, Dynamic finite element analysis

\section{INTRODUCTION}

Masonry structures are one of the most commonly used building types in developing countries due to their inexpensiveness and accessibility as construction material. While masonry structure is intrinsically strong under gravitational load, it is weak against lateral forces such as an earthquake. Historically, the collapses of structures due to earthquakes account for almost $75 \%$ of the earthquake fatalities [1], and the collapse of masonry structures accounts for a large number of fatalities among them [2]. Masonry structures are still commonly used in earthquakeprone areas in developing countries because they are inexpensive and easy to construct. To reduce causalities due to the collapse of the masonry structures in developing countries, reinforcing the masonry structure is deemed necessary.

The usage of steel reinforcing bars for masonry structures is one of the most commonly used reinforcement methods in developed countries. Its application, however, is not always easy in developing countries due to the lack of resources and knowledge [3]. Consequently, reinforcement methods for masonry structures that are costefficient and easy to implement are much preferable.

In the past several years, many kinds of research on the reinforcement of masonry structures have been conducted. Among them, reinforcement methods by utilizing vertical steel ties [4], fiberreinforced plastic [5], tires [6] (as a tensioned material), and PP bands [7] are some of the examples which are easy to implement, and utilizing readily available materials, but require additional reinforcing material.

In this study, a reinforcing method that does not require reinforcing materials is considered for masonry structures. The method uses interlocking bricks instead of regular rectangular bricks. Interlocking bricks are bricks that utilize an interlocking system to reinforce against loading. When a joint failure occurs in a rectangular brick wall, the external force is resisted by only friction force (Fig.1(a)). In comparison, when a joint failure occurs in an interlocking brick wall, the external force is resisted by both friction force and the interlocking mechanism (Fig. 1(b)). By utilizing this concept, the interlocking brick is assumed to be able to reinforce masonry structures. Additional benefits of this method are that it does not require additional reinforcement material and is easy to implement.

Researches on the performance of interlocking bricks against earthquake have been conducted [811]. Research [11] showed that interlocking bricks could reinforce masonry walls against the in-plane static lateral load. However, since earthquakes are

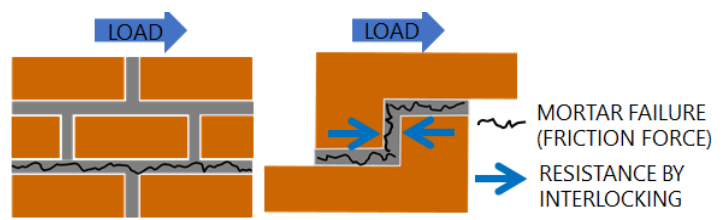

(a)Rectangular brick (b)Interlocking brick

Fig.1 Difference in the resistance mechanism against lateral load between rectangular and interlocking brick walls 

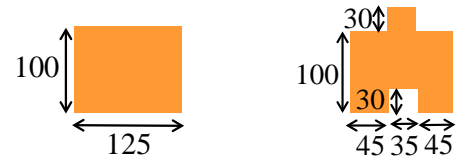

Rectangular brick Lego-shaped brick

(a)Out-of-plane wall model

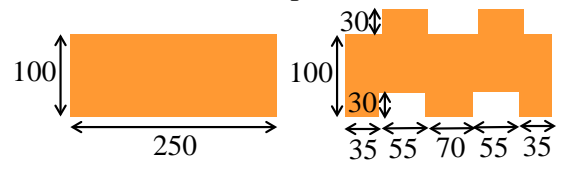

Rectangular brick Lego-shaped brick

(b) Out-of-plane two-wall model

Fig.2 Assumed bricks (unit: mm)

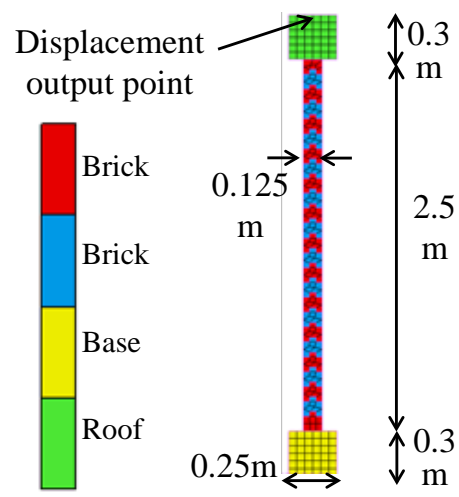

(a)Out-of-plane wall model

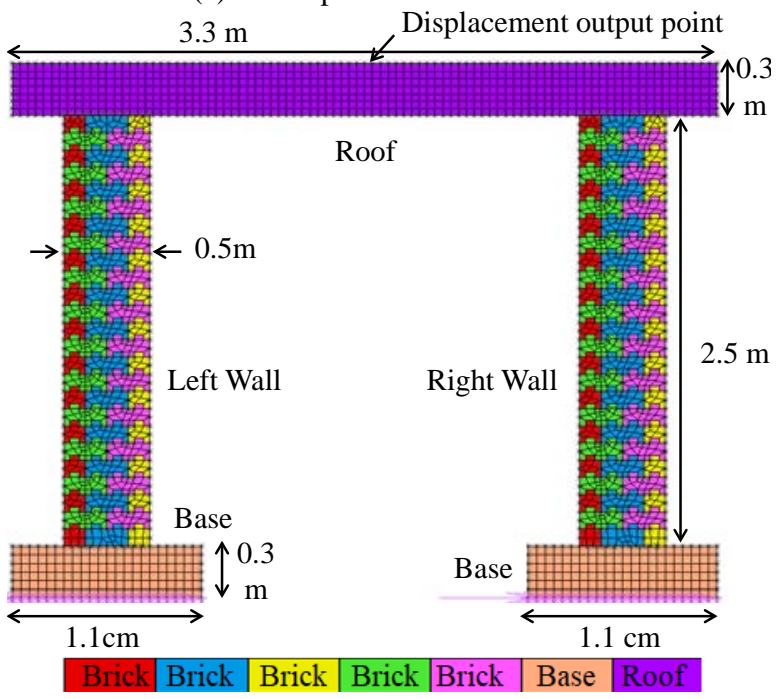

(b) Out-of-plane two-wall model

Fig.3 Wall models made of lego-shaped bricks
(a)Rectangular brick

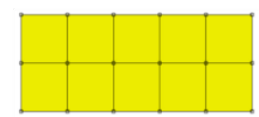

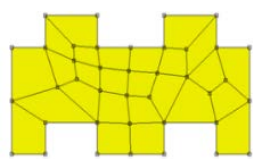

(b)Lego-shaped brick
Fig.4 Division of brick into elements

dynamic loads, which also act in the out-of-plane direction, research on interlocking bricks' performance against dynamic lateral load in the outof-plane direction is necessary. However, such researches have not been conducted yet. With this background, this paper aims to investigate the performance of interlocking bricks in resisting outof-plane lateral excitation through dynamic finite element analysis.

\section{ANALYSIS CONDITIONS}

\subsection{Analysis Models}

In this study, two-dimensional dynamic analysis of masonry walls was performed using the generalpurpose finite element analysis software, MSC Marc [12].

There are four analysis models, two one-wall models, and two two-wall models. Two one-wall models and two two-wall models are divided into a rectangular brick type and an interlocking wall type.

Dimensions of bricks for each model are shown in Fig. 2. Rectangular bricks and lego-shaped bricks are considered. In past research [8-11], I-shaped bricks and wave-shaped bricks were considered, which have the interlocking effect only in one direction. Since earthquake acts both in the in-plane and out-of-plane directions, the lego-shaped brick was considered since they have the interlocking effect in both directions.

Two-dimensional analysis models are shown in Fig.3. Analysis models in Fig. 3 are examples of the interlocking brick walls. Analysis models for the rectangular brick wall were also created similarly.

Fig. 3(a) is a thin wall model with only one brick in the horizontal direction, and Fig. 3(b) is a twowall model with thicker walls. Assuming that the roof is supported by more than two walls in actual buildings, Fig.3(b) is considered.

The lego-shaped bricks are only used for the wall part. The protrusions of bricks on top of the wall were neglected, and the top surface was smoothed. In the same way, holes for engaging with protrusions at the bottom of the wall are neglected.

Actual buildings have three-dimensional shapes and show three-dimensional behavior. However, this study used two-dimensional analysis to investigate the out-of-plane vibration since the wall is weak against the out-of-plane direction.

All nodes at the bottom are fixed in both horizontal and vertical directions. The horizontal inertia force was applied to all free nodes when inputting horizontal acceleration.

Fig. 4 indicates the example of how the rectangular and interlocking bricks are divided into elements. Plane strain 4-noded elements were utilized. The size of each element for the rectangular brick, roof, and steel elements is $5 \mathrm{~cm} \times$ $5 \mathrm{~cm}$. The lego-shaped brick was also divided into elements of a similar size.

Material properties are shown in Table 1(a). The base and roof are made of steel so that their deformation can be neglected. 
Table 1 Analysis Parameters

(a)Material properties

\begin{tabular}{ccc}
\hline Value & Brick & Base \& Roof \\
\hline Density $\left(\mathrm{kg} / \mathrm{mm}^{3}\right)$ & $1.9 \times 10^{-6}$ & $8.05 \times 10^{-6}$ \\
Young Modulus $\left(\mathrm{N} / \mathrm{mm}^{2}\right)$ & 19,000 & 200,000 \\
Poisson's Ratio & 0.216 & 0.2 \\
\hline
\end{tabular}

(b)Mortar properties

\begin{tabular}{cc}
\hline Value & Mortar \\
\hline Tensile strength $\left(\mathrm{N} / \mathrm{mm}^{2}\right)$ & 0.3 \\
\hline Shear strength $\left(\mathrm{N} / \mathrm{mm}^{2}\right)$ & 0.3 \\
\hline Friction coefficient & 0.463 \\
\hline
\end{tabular}

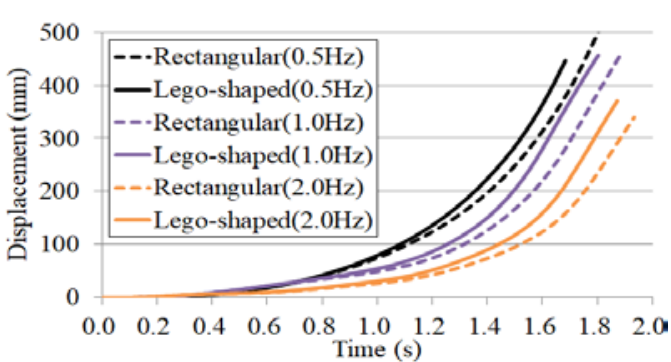

Fig.5 Horizontal displacement history of one-wall models

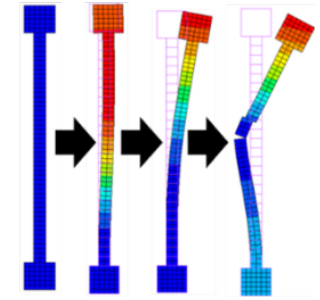

(a)Rectangular brick

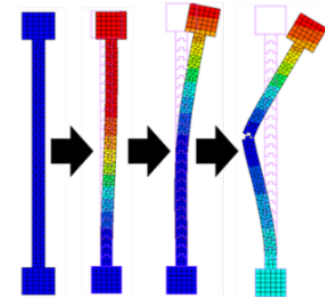

(b)Lego-shaped brick
Fig.6 Deformation process of one-wall models $(1 \mathrm{~Hz})$

\subsection{Modeling of Failure}

It is considered that bricks are connected with mortar, and failure occurs at the mortar. Therefore, it was set that all bricks do not shear the nodes with other bricks. The bricks are glued with the adjacent bricks at the interface. The bricks move together with the adjacent bricks unless the glue does not get broken.

The mortar failure is modeled by the failure of the glue between elements. When the normal or shear stress at the interface between elements exceeds the tensile or shear strength, the glue is broken. After the failure, only compression and friction force acts between the elements. Mortar properties are shown in Table 1(b).

\subsection{Input Accelerations}

Sinusoidal accelerations with the amplitude of 20 gal and 120gal were input to the one-wall models and two-wall models in the out-of-plane direction.
Three frequencies, $0.5 \mathrm{~Hz}, 1.0 \mathrm{~Hz}$, and $2.0 \mathrm{~Hz}$, were used to investigate the performances under various frequencies.

The single-step Houbolt time integration method was used $\left(\gamma_{1}=1.5\right.$ and $\left.\gamma=-0.5\right)$.

\section{RESULTS OF NUMERICAL ANALYSIS}

\subsection{Overview}

The analysis results of out-of-plane lateral excitation are discussed. The performance of the lego-shaped brick wall and the rectangular brick wall are compared concerning the displacement history and deformation process.

\subsection{One-wall Models}

In one-wall models, sinusoidal accelerations of 20 gal with the frequency of $0.5 \mathrm{~Hz}, 1.0 \mathrm{~Hz}$, and $2.0 \mathrm{~Hz}$ were input in the horizontal direction of Fig. 3(a).

The horizontal displacement history for all frequency cases is shown in Fig. 5. The walls are vulnerable, and both models collapsed by the sinusoidal acceleration of even 20 gal irrespective of the frequency.

Since all models collapsed and showed similar behavior, the deformation process of the $1.0 \mathrm{~Hz}$ case is shown in Fig. 6 as an example. The walls got fractured at the middle height and broken into two parts. The color contour of Fig. 6 indicates the horizontal displacement. The red color is the maximum displacement, and the blue color is the minimum displacement. The right side is the positive direction of the displacement. The top of the model has the largest displacement. After the walls are broken into two pieces, the middle part showed the minimum displacement since it moved to the left.

Since there is only one brick in the out-of-plane direction, the interlocking effect is small and could not resist the out-of-plane excitation.

\subsection{Two-wall Models}

\subsubsection{Dominant frequency}

The one-wall model was too thin and got easily fractured even with a slight acceleration of 20gal. It was found the interlocking system does not work in such cases where the wall itself is vulnerable.

Next, two-wall models are analyzed. Their wall width is $50 \mathrm{~cm}$, which is four times thicker than that of the one-wall models. Furthermore, the roof is supported by two walls, which means more stability. It is considered that the two-wall models are more realistic since the roof weight is usually supported by more than two walls in the actual buildings. 


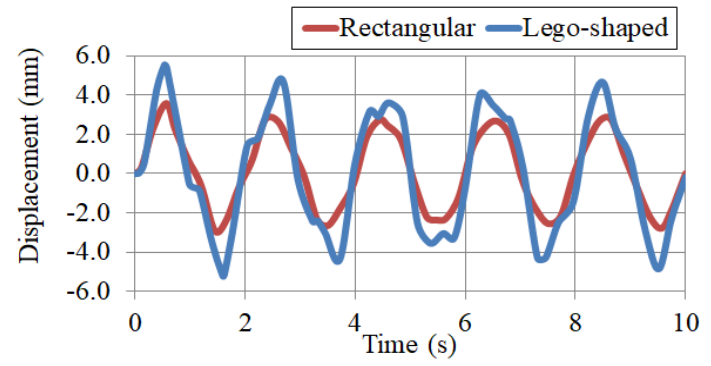

Fig.7 Horizontal displacement history of two-wall models $(0.5 \mathrm{~Hz})$

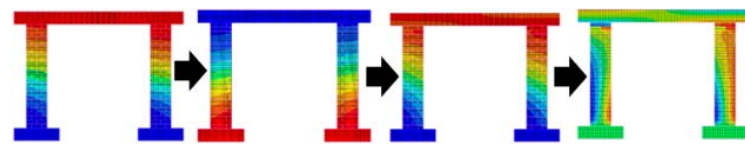

(a)Rectangular $(0.5,1.5,2.5$, and $3.5 \mathrm{sec}$, from the left)

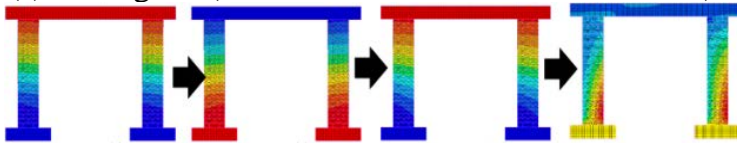

(b)Lego-shaped $(0.5,1.5,2.5$, and $3.5 \mathrm{sec}$, from the left)

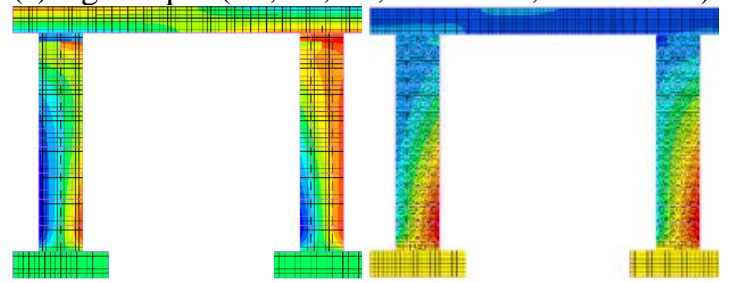

(c)Failure observed at the (d)No failure observed to top of both walls of the the lego-shaped brick rectangular brick wall wall $(3.5 \mathrm{sec})$

(3.5 sec)

Fig. 8 Deformation process and horizontal displacement contour of two-wall models for $0.5 \mathrm{~Hz}$ (red: maximum, blue: minimum)

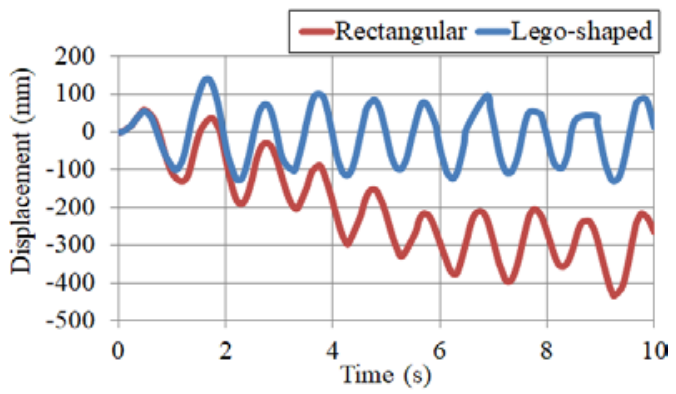

Fig.9 Horizontal displacement history of two-wall models for $1.0 \mathrm{~Hz}$

The dominant frequencies of the two-wall models were obtained from their free vibration responses. The models were excited by inputting an impulsive acceleration, where the acceleration amplitude for the first $0.01 \mathrm{sec}$ is 1 gal and that for the left $4.99 \mathrm{sec}$ is 0 gal. Then the displacement histories at the top center of the roof were Fourier transformed, and the predominant frequencies were read. The dominant frequencies of both two-wall models were about $1.25 \mathrm{~Hz}$.
Therefore, the input acceleration cases were divided into three categories, the case lower than the dominant frequency $(0.5 \mathrm{~Hz})$, the case around the dominant frequency $(1.0 \mathrm{~Hz})$, and the cases higher than the dominant frequency $(2.0 \mathrm{~Hz})$.

When the sinusoidal acceleration of 20gal is input, both models experienced no failure. When no failure occurs, no difference is observed between the rectangular and lego-shaped brick models since there is no need for the interlocking system to work. Therefore, the lateral load was increased up to 120 gal, where a noticeable performance difference between the two models was observed.

\subsubsection{Result for $0.5 \mathrm{~Hz}$}

The horizontal displacement history for $0.5 \mathrm{~Hz}$ is shown in Fig. 7. The lego-shaped brick wall showed a slightly larger deformation than the rectangular brick wall, but its value is small.

The deformation process and displacement contour for $0.5 \mathrm{~Hz}$ are shown in Fig. 8(a)(b). Both models showed similar behavior until $2.5 \mathrm{sec}$, but the difference occurred at $3.5 \mathrm{sec}$. The enlarged figures at $3.5 \mathrm{sec}$ are shown in Fig.8(c)(d).

Non-smoothness of displacement contour indicates the occurrence of failure. As shown in Fig. 8(c), the rectangular brick wall experienced failure around the top of both walls. In contrast, the legoshaped brick wall shows a smooth displacement contour (Fig.8(d)), and no failure was observed. It was found that the interlocking system could avoid failure around the top of the walls.

\subsubsection{Result for $1.0 \mathrm{~Hz}$}

The horizontal displacement history for $1.0 \mathrm{~Hz}$ is shown in Fig. 9. Compared to the result for 0.5 $\mathrm{Hz}$, both models showed more significant horizontal displacement. The horizontal displacement of the rectangular brick wall model was gradually shifted to the negative direction, which means the roof moved to the left.

The deformation process and displacement contour for $1.0 \mathrm{~Hz}$ are shown in Fig. 10(a)(b). Fig. 10(a) shows that the sliding movement of the roof occurred in the rectangular brick wall since the horizontal cracks occurred on the upper walls. On the other hand, no sliding displacement occurred in the lego-shaped brick wall owing to the interlocking system (Fig. 10(b)).

The enlarged figures at $2.2 \mathrm{sec}$ are shown in Fig.9(c)(d). As shown in Fig. 9(c), the rectangular brick wall experienced horizontal cracks at the top of both walls and diagonal cracks at the bottom of both walls. In contrast, the lego-shaped brick wall experienced no diagonal cracks at the bottom of the walls. Mortar failure occurred at the top of both walls, but no sliding between the walls and the roof occurred due to the interlocking system. 


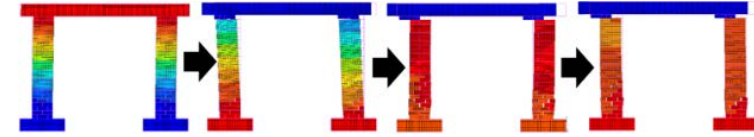

(a)Rectangular $(0.4,1.0,1.6$, and $2.2 \mathrm{sec}$, from the left)

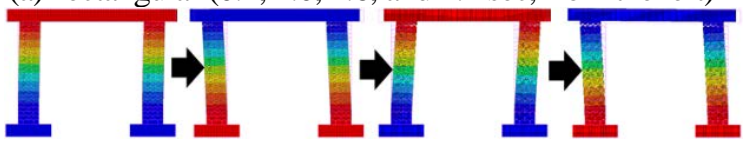

(b)Lego-shaped $(0.4,1.0,1.6$, and $2.2 \mathrm{sec}$, from the left)

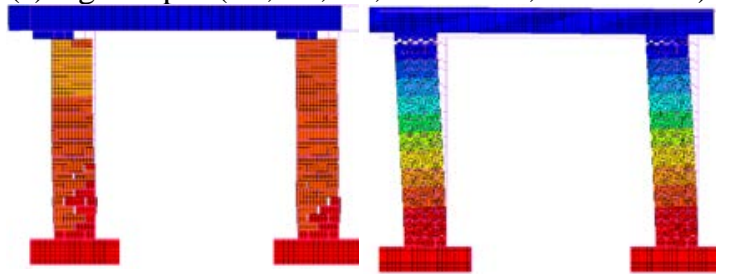

(c)Failure observed at the (d)No failure observed to top of both walls of the the lego-shaped brick rectangular brick wall wall $(2.2 \mathrm{sec})$

(2.2 sec)

Fig. 10 Deformation process and horizontal displacement contour of two-wall models for $1.0 \mathrm{~Hz}$ (red: maximum, blue: minimum)

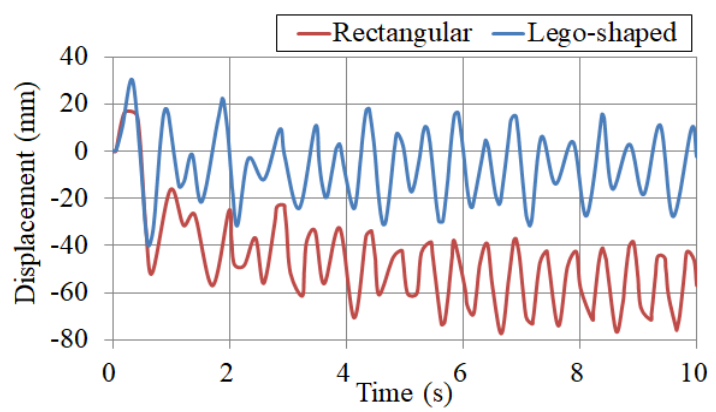

Fig.11 Horizontal displacement history of two-wall models for $2.0 \mathrm{~Hz}$

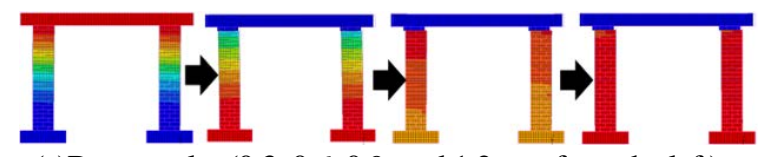

(a)Rectangular $(0.3,0.6,0.9$, and $1.2 \mathrm{sec}$, from the left)

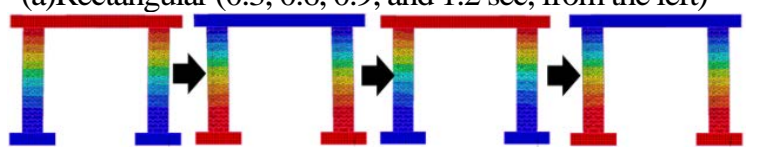

(b)Lego-shaped $(0.3,0.6,0.9$, and $1.2 \mathrm{sec}$, from the left)

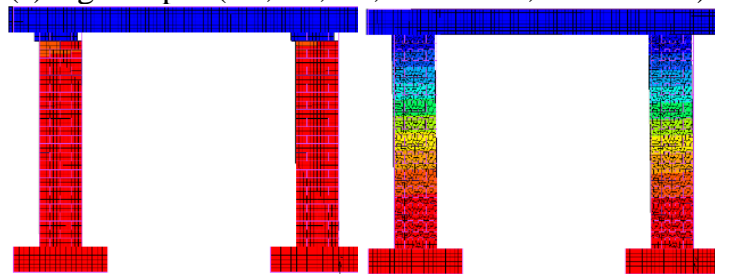

(c)Failure observed at the (d)No failure observed to top of both walls of the the lego-shaped brick rectangular brick wall wall $(1.2 \mathrm{sec})$

(1.2 sec)

Fig. 12 Deformation process and horizontal displacement contour of two-wall models for $2 \mathrm{~Hz}$ (red: maximum, blue: minimum)

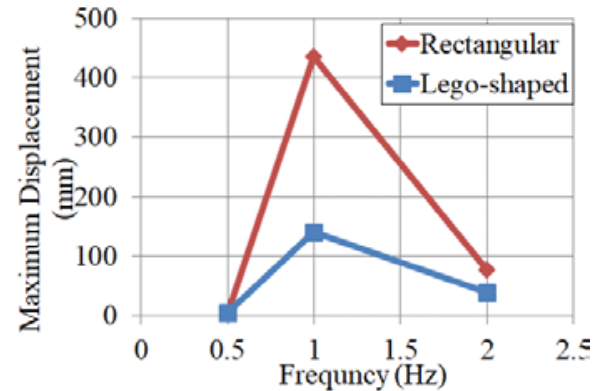

Fig. 13 Comparison of the maximum displacement for two-wall models

\subsubsection{Result for $2.0 \mathrm{~Hz}$}

The horizontal displacement history for $2.0 \mathrm{~Hz}$ is shown in Fig. 11. They showed a similar tendency to the case of $1.0 \mathrm{~Hz}$ even though the horizontal displacement of $2.0 \mathrm{~Hz}$ is small. The horizontal displacement of $2.0 \mathrm{~Hz}$ is smaller since $2.0 \mathrm{~Hz}$ is farther from the dominant frequency of $1.25 \mathrm{~Hz}$ than $1.0 \mathrm{~Hz}$.

The deformation process and displacement contour for $2.0 \mathrm{~Hz}$ are shown in Fig. 12(a)(b). Fig. 12(a) shows that the sliding movement of the roof occurred in the rectangular brick wall since the horizontal cracks occurred on the upper walls. On the other hand, no sliding displacement occurred in the lego-shaped brick wall, and horizontal displacement could be kept small due to the interlocking system (Fig. 12(b)).

The enlarged figures at $1.2 \mathrm{sec}$ are shown in Fig.12(c)(d). In the rectangular brick wall model, the upper part of both walls got fractured. In the lego-shaped brick wall model, no failure occurred, and the model deformed as a united body.

\subsection{Summary}

The interlocking system did not work for thin one-wall models, while it worked for thick two-wall models.

The comparison of the maximum displacement for two-wall models is shown in Fig. 13. In all frequencies, the rectangular brick wall model showed larger displacement than the lego-shaped brick wall model.

The displacement suppression effect of the interlocking system was the largest at $1.0 \mathrm{~Hz}$ when both models had the largest displacement due to resonance, and the displacement of the lego-shaped brick wall was about $1 / 3$ of that of the rectangular brick wall.

In the case of $2.0 \mathrm{~Hz}$, the large displacement response and failure occurred to the rectangular brick wall. In contrast, no failure occurred, and displacement was kept small in the lego-shaped brick wall since the interlocking system worked.

In the case of $0.5 \mathrm{~Hz}$, the displacement response was small and slight failure occurred to the 
rectangular brick wall. Since there was not much room for the interlocking system to work, the displacement suppression effect of the lego-shaped brick wall was small. No damage occurred in the lego-shaped brick wall, and the interlocking effect was observed in damage prevention.

It could be concluded that the lego-shaped brick wall shows better performance in resting the lateral dynamic load even in the out-of-plane direction when the wall is not too thin.

\section{CONCLUSION}

In this study, the performance of the interlocking brick walls under lateral dynamic load in the out-of-plane direction was investigated using the 2-dimensional finite element analysis. The legoshaped brick was chosen, and its performance was compared to the rectangular brick. Two models were compared. The first is a one-wall model of a single thin wall supporting a roof, and the second is the two-wall model of two thicker walls supporting the roof. Sinusoidal acceleration was input to the models with three frequencies of $0.5 \mathrm{~Hz}, 1.0 \mathrm{~Hz}$, and $2.0 \mathrm{~Hz}$.

In the one-wall models, both rectangular and interlocking brick wall models quickly got fractured and collapsed irrespective of the input frequencies. It was found that the interlocking system does not work for a thin wall subjected to the out-of-plane loading since the wall itself was vulnerable.

In the case of thick two-wall models, the interlocking system worked. The rectangular brick wall got failure, but the failure was avoided, and displacement response was kept small in the interlocking brick wall. When the displacement response is small, the effect of the interlocking system is small. As the displacement response was larger, the interlocking system worked, and the displacement suppression effect was noticeable. The interlocking system caused the brick wall to deform together as a unit, eliminating the unwanted sliding movement due to failure.

It was confirmed that the lego-shaped brick walls showed a better performance in resisting the out-of-plane dynamic load due to the interlocking system if the wall is thick enough to exert the interlocking effect.

\section{REFERENCES}

[1] OCHA (Office for the Coordination of Humanitarian Affairs): www.unocha.org. [Accessed April 16, 2021].

[2] Coburn, A., and Spence, R., Earthquake protection 2nd Edition, Chichester, John Wiley and Sons, 2002.

[3] McWilliams, H., and Griffin, C.T, A critical assessment of concrete and masonry structures for reconstruction after seismic events in developing countries, Structures and Architecture: Concepts, applications, and challenges, Proceedings of the Second International Conference on Structures and Architecture, CRC Press, 2013, pp. 857-864.

[4] Darbhanzi, A., Marefat, S., and Khanmohammadi, M., Investigation of in-plane seismic retrofit of unreinforced masonry walls using vertical steel ties, Construction and Building Materials, Vol. 52, 2014, pp. 122-129.

[5] Marcari, G., Manfredi, G., Prota, A., and Pecce, M., In-plane shear performance of masonry panels strengthened with FRP, Composites: Part B, Vol. 38, 2007, pp. 887-901.

[6] Turer, A., and Golalm, M., Scrap tire as lowcost post-tensioning material for masonry strengthening, Materials and Structures, Vol. 41, 2011, pp. 1345-1361.

[7] Sathiparan, N., Sakurai, K., and Meguro, K., Experimental study of PP-band retrofitted masonry structure made of shapeless stone. SEISAN-KENKYU, Vol. 61, Issue 6, 2009, pp. 1051-1054.

[8] Sanada, Y., Nakamura, Y., Yamauchi, N., and Akano, Y., Seismic performance of masonry walls using interlocking units, Proceedings of the First European conference on earthquake engineering and seismology, Paper No. 508, 2006.

[9] Furukawa, A., Kimura, S., and Kiyono, J., Study on failure mechanism and strength increment effect of masonry walls made of interlocking bricks, Journal of Japan Society of Civil Engineers, Vol. 74, No. 4, 2018, pp. I_699I_711 (in Japanese).

[10] Furukawa, A., Masuda, K., Daru, G.T.S., and Kiyono, J., Shape effect of interlocking blocks on force-displacement relationship and failure behavior of masonry wall, Journal of Structural Engineering, Vol. 64A, 2018, pp. 241-249 (in Japanese).

[11] Furukawa, A., Prasetyo, J.J., Kiyono, J., Failure process and load-displacement relationship of rectangular block and interlocking block walls during in-plane lateral loading, Journal of Japan Society for Natural Disaster Science, Vol. 38, 2019, pp.25-41.

[12] MSC Software Corporation: Marc, www.mscsoftware. Com/product/marc. [Accessed April 16, 2021.]

Copyright (C) Int. J. of GEOMATE All rights reserved, including making copies unless permission is obtained from the copyright proprietors. 